\title{
Early onset sepsis calculator implementation is associated with reduced healthcare utilization and financial costs in late preterm and term newborns
}

\author{
Niek B. Achten ${ }^{1,2}$ (D) Douwe H. Visser ${ }^{3}$. Ellen Tromp ${ }^{4}$ - Wim Groot ${ }^{5}$ • Johannes B. van Goudoever ${ }^{2}$ • Frans B. Plötz ${ }^{1,2}$
}

Received: 21 August 2019 / Revised: 11 October 2019 / Accepted: 15 October 2019 / Published online: 2 January 2020

(C) The Author(s) 2020

\begin{abstract}
The neonatal early onset sepsis (EOS) calculator is a novel tool for antibiotic stewardship in newborns, associated with a reduction of empiric antibiotic treatment for suspected EOS. We studied if implementation of the EOS calculator results in less healthcare utilization and lower financial costs of suspected EOS. For this, we compared two single-year cohorts of hospitalizations within 3 days after birth in a Dutch nonacademic teaching hospital, before and after implementation of the EOS calculator. All admitted newborns born at or after 35 weeks of gestation were eligible for inclusion. We analyzed data from 881 newborns pre-implementation and 827 newborns post-implementation. We found significant reductions in EOS-related laboratory tests performed and antibiotic days, associated with implementation of the EOS calculator. Mean length of hospital stay was shorter, and EOS-related financial costs were lower after implementation among term, but not among preterm newborns.

Conclusion: In addition to the well-known positive impact on antibiotic stewardship, implementation of the EOS calculator is also clearly associated with reductions in healthcare utilization related to suspected EOS in late preterm and term newborns and with a reduction in associated financial costs among those born term.
\end{abstract}

What is Known:

- The early-onset sepsis (EOS) calculator is a novel tool for antibiotic stewardship in newborns, associated with a reduction in empiric antibiotic treatment for suspected EOS.

What is New:

- In newborns at risk for EOS, EOS calculator implementation is associated with a significant reduction in laboratory investigations related to suspected EOS and significantly shorter stay in those born term.

- EOS calculator implementation in term newborns is associated with a mean reduction of $€ 207$ in costs for EOS-related care per admitted newborn.

Keywords Costs $\cdot$ Early-onset sepsis $\cdot$ EOS calculator $\cdot$ Healthcare utilization $\cdot$ Newborn

Communicated by Patrick Van Reempts

Niek B. Achten

niek.achten@gmail.com

Douwe H. Visser

d.h.visser@amsterdamumc.nl

Ellen Tromp

ellen.tromp@kpnmail.nl

Wim Groot

w.groot@maastrichtuniversity.nl

Johannes B. van Goudoever

h.vangoudoever@amsterdamumc.nl

Frans B. Plötz

fbplotz@tergooi.nl
1 Department of Pediatrics, Tergooi Hospitals, Rijksstraatweg 1, 1261 AN, Blaricum, The Netherlands

2 Amsterdam UMC University of Amsterdam, Vrije Universiteit, Department of Pediatrics, Emma Children's Hospital, Amsterdam, Netherlands

3 Amsterdam UMC University of Amsterdam, Vrije Universiteit, Department of Neonatology, Emma Children's Hospital, Amsterdam, Netherlands

4 Department of Epidemiology and Statistics, St Antonius Hospital, Nieuwegein, Netherlands

5 Department of Health Services Research, School for Public Health and Primary Care, Faculty of Health, Medicine and Life Sciences, Maastricht University, Maastricht, Netherlands 


\section{Abbreviations \\ CBC Complete blood count \\ CRP C-reactive protein \\ EOS Early-onset sepsis}

\section{Methods}

\section{Study setting, design. and patients}

This single-center before-after EOS calculator implementation study was conducted in a Dutch nonacademic teaching hospital with a mother-child unit and a neonatal ward. The hospital provides care up to level II special care for stable or moderately ill newborns[11] and admits newborns for various reasons. Our study compared two single-year birth cohorts. We screened all newborns born in our hospital from January 1, 2014, through December 31, 2014 (pre-implementation cohort), and from April 1, 2016, through March 31, 2017 (post-implementation cohort) (Fig. 1). We evaluated all births at or after 35 weeks of gestation and included newborns admitted for pediatric care within 3 days after birth. The current study is a post hoc analysis of our implementation study, which focused on the rate of empiric antibiotic treatment in the entire birth cohort [3]. For the current analysis, we focused on admitted newborns, because it is the population susceptible to EOS care utilization and associated costs.

\section{Clinical practice before and after implementation of the sepsis calculator}

Before implementation, newborns born at our hospital were screened for maternal risk factors and clinical symptoms by the attending staff from the mother-child unit. Maternal EOS risk factors warranting pediatric evaluation included prolonged rupture of membranes (more than 18 hours), maternal fever $\left(38{ }^{\circ} \mathrm{C}\right.$ or higher), prematurity, and positive maternal GBS status. Newborns requiring evaluation or care by pediatric staff for any reason were admitted for hospital care, either at the mother-child unit or neonatal ward. Newborns not admitted for hospital care accompanied mother in the motherchild unit or were discharged home.

Before implementation, a newborn at risk for EOS was assigned observation on vital signs or treatment with empiric antibiotics. This arbitrary decision was made by the attending physician, based on the combination of maternal EOS risk factors, physical examination, and/or results of complete blood count (CBC) and C-reactive protein (CRP). Within the study population, prematurity was defined as birth at 35 to 37 weeks' gestation. If born between 35 weeks and 35 weeks and 6 days of gestation, newborns were always admitted to the neonatal ward. Without other risk factors or clinical symptoms, prematurity alone was not a reason to start empiric antibiotic treatment per se. If default empiric antibiotic therapy was started, it consisted of intravenous gentamicin and amoxicillin, followed by intravenous amoxicillin/clavulanic acid after 72 hours if not discontinued. Before the start of antibiotic treatment, blood was drawn for a CBC, CRP, and blood culture. A gentamicin serum concentration was determined and 


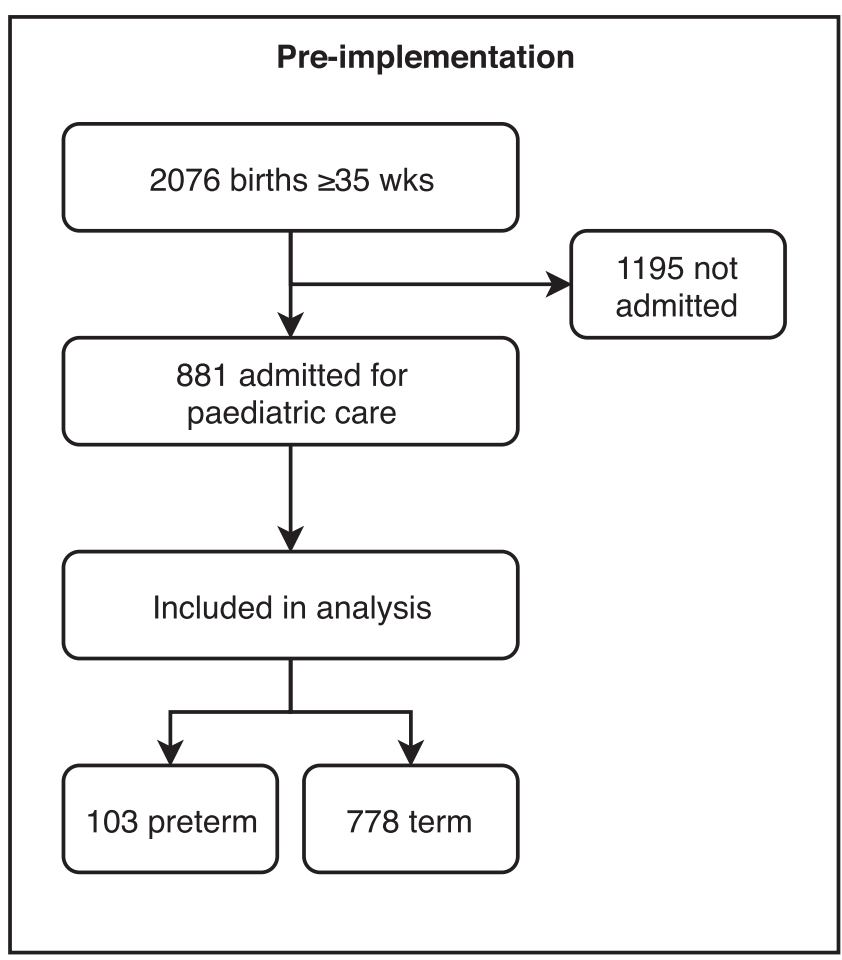

Fig. 1 Study inclusion. Flowchart of study inclusion process

repeated if necessary. CBC and CRP were repeated after 4872 hours of antibiotic treatment. In case of a negative blood culture after 3 days of treatment, antibiotic treatment was either stopped, or continued for clinical reasons, per discretion of the attending physician. In case of a positive blood culture, antibiotic treatment was continued for at least 7 days from start. If continued despite a negative culture, treatment was continued for 7 days.

After implementation of the EOS calculator, each birth was screened for maternal EOS risk factors and clinical symptoms, as before implementation. In case of 1 or more maternal EOS risk factors or if the newborn showed any clinical signs of EOS, prompt clinical evaluation of the newborn followed, using the EOS calculator. Based on the EOS risk calculation, in our hospital, two options were possible: either start empiric antibiotics at the neonatal ward or perform routine control of vital parameters every 3 hours at the maternal-child or neonatal ward for at least 24 hours. The EOS sepsis calculator recommendation obtaining a blood culture without starting antibiotic treatment was incongruent with our practice, and this recommendation was therefore followed by the second option. In case of antibiotic treatment, treatment protocol was equal to before implementation, as described above. Treating physicians were free to deviate from the recommendation by the calculator.

\section{Data collection and outcomes}

Data were obtained electronically from the clinical, pharmaceutical, and financial hospital registration and billing

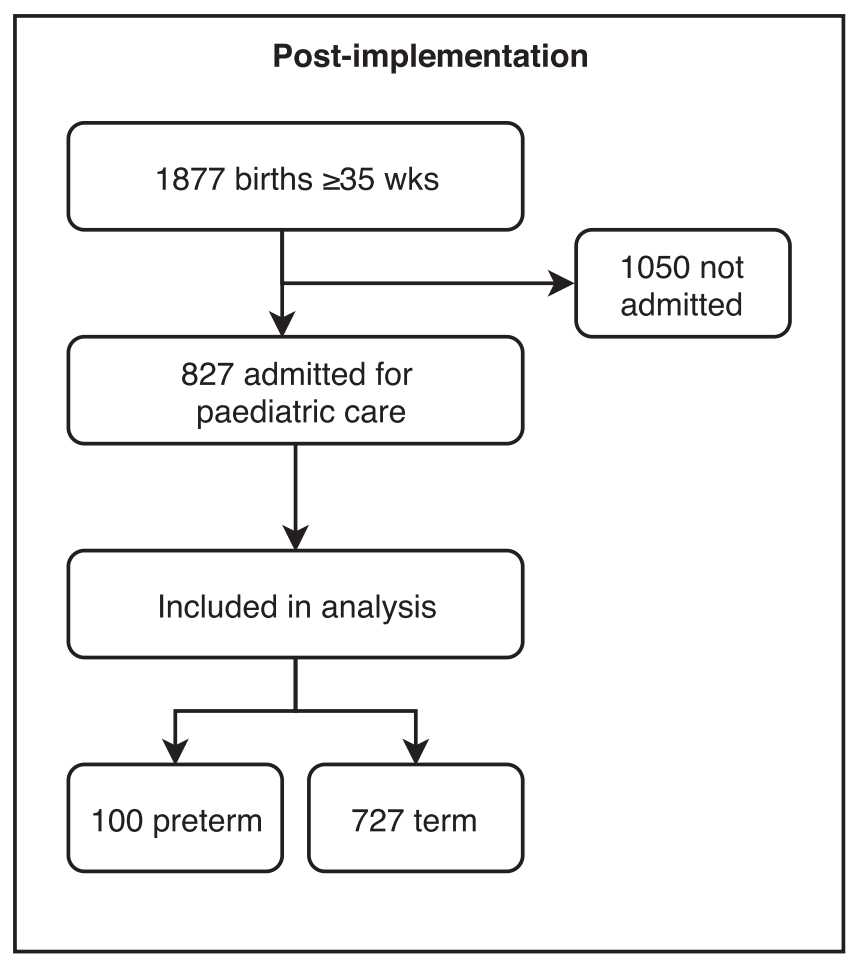

systems. For the first outcome, EOS-related healthcare utilization, we included three groups of clinical outcomes: outcomes related to hospital length of stay, outcomes related to relevant laboratory tests (blood cultures; complete or partial blood counts; CRP; gentamicin serum concentration), and outcomes regarding (empiric) antibiotic treatment for EOS (start of antibiotic treatment, number of antibiotic days). For the second outcome, defined as financial costs related to EOS healthcare, we retrieved for each group of clinical outcomes the related costs from the hospital billing administration and calculated the combined financial cost. Costs associated with EOS-related antibiotics were calculated using costs for antibiotics in the protocol for suspected EOS described above. Because in-house billing costs were not different between the mother-child unit and the neonatal ward, these costs were not separated. To insulate our analysis from temporal cost changes during the study timeframe, we used 2017 in-house billing costs throughout analysis for both cohorts. Costs in this study represent actual cost of care, rather than final billing charges.

\section{Statistical analysis}

Data from newborns hospitalized before implementation were compared with data from newborns hospitalized after implementation. Subgroup analyses were performed for term and preterm newborns. We also compared newborns with and without antibiotic treatment. Categorical variables were reported as (relative) frequencies with and compared with chi- 
square analysis. Continuous variables were reported as means with standard deviation (SD) to provide meaningful outcome measures and compared using Welch two-sample t-test, which is appropriate for skewed distributions [12-14]. All analyses were performed using R version 3.5.2 (R Foundation, Vienna, Austria).

\section{Results}

\section{Inclusions}

The year after implementation involved 1877 births at or after 35 weeks of gestation of which 827 (44.1\%) were admitted for pediatric care in the first three days after birth, compared with 2076 births and 881 (42.4\%) admissions before implementation. All admitted newborns were included in the analysis. Fifty of 827 (6.0\%) admitted newborns were started on empiric antibiotics for suspected EOS after implementation, compared with 100 of 881 (11.4\%) before implementation (P < $0.001)$. The rate of prematurity was comparable in both cohorts $(12.1 \%$ after versus $11.7 \%$ before implementation, $\mathrm{P}=$ $0.798)$.

\section{EOS healthcare utilization}

Healthcare utilization was assessed for three clinical outcome groups (Table 1 and Fig. 2). Mean length of stay did not differ significantly between the two cohorts in the overall study sample but was 0.37 days shorter after implementation among term newborns specifically, $(\mathrm{P}=0.005)$. We found a significant reduction in mean number of EOS-related laboratory tests per newborn after implementation $(\mathrm{P}<0.001$, Table 1$)$, including fewer blood cultures, blood counts, CRP, and gentamicin serum concentration tests $(\mathrm{P} \leq 0.001)$. The use of antibiotic treatment was significantly lower after implementation (number of antibiotic days, $\mathrm{P}=0.009$ ). Start of empiric antibiotics in at-risk newborns, independent of implementation, was associated with significant more EOS healthcare utilization (Table 2).

\section{EOS care financial costs}

Mean costs related to length of stay did not differ significantly between cohorts in the overall population but were significantly lower after implementation in the subpopulation of term newborns (Table 1 and Fig. 2). Mean costs associated with EOS-related laboratory tests and the use of empiric antibiotics were significantly lower after implementation $(36.8 €$ vs 24.9 $€ ; \mathrm{P}<0.001$ and $1.54 €$ vs $0.96 € ; \mathrm{P}=0.008$, respectively). Mean combined cost associated with EOS-related care per included newborn did not differ between cohorts in the overall population but were significantly lower after implementation among term newborns specifically (2248€ vs 2041€; P = 0.020 ). Combined mean costs were dominated by costs related to length of stay, which accounted for $98.5 \%$ of combined costs after implementation and $99.0 \%$ before implementation.

A total of four culture-confirmed EOS cases occurred during the study period, two before and two after implementation. The mean combined costs associated with EOS-related care for these cases were $€ 7415$ per newborn. Culture-confirmed EOS represented $0.7 \%$ of total cost associated with EOSrelated care in the entire study period.

\section{Discussion}

This before-after study evaluated the effect of implementation of the EOS calculator on EOS-related healthcare utilization and the related financial costs in late preterm and term newborns. Implementation of the EOS calculator was associated with a significant reduction in laboratory investigations for suspected EOS and lower costs associated with these tests. In addition, we found that significant reductions in length of stay or overall EOS-related hospital costs associated with implementation of the EOS calculator were limited to the term newborn population.

Implementation of the EOS calculator was associated with fewer antibiotic days. Fewer newborns were started on antibiotics, but the duration of an antibiotic course was similar after implementation [3]. Therefore, observation of fewer antibiotic days is most likely due to fewer cases of "rule out sepsis" rather than fewer extended courses of antibiotics. Because each instant of blood collection and insertion of peripheral catheter for administration of antibiotics entails a painful procedure and a risk of infection, the reductions and antibiotic days and EOS-related laboratory tests imply a reduction in clinical burden and hazards. This effect may be emphasized downstream, as investigations like repeated CRP for suspected EOS lead to further investigations and longer treatment [15].

Our study shows that length of stay is the primary driver for costs in this at-risk population and that newborns treated with antibiotics have more than twofold higher EOS-related costs than those not treated (Table 2). Despite a clear reduction in antibiotic treatment in both term and preterm newborns after EOS calculator implementation, reductions in length of stay and costs after EOS calculator implementation were limited to term newborns. We suggest two explanations for the lack of clear reductions in length of stay of preterm newborns. First, the number of preterm newborns was relatively small, limiting statistical power to detect reductions in length of stay in this subgroup. Second, both prematurity in itself and related neonatal problems such as feeding difficulties warrant hospital stay, regardless of the decision to treat for EOS. 
Table 1 EOS healthcare utilization and associated costs before and after EOS calculator implementation

\begin{tabular}{|c|c|c|c|}
\hline & $\begin{array}{l}\text { Before implementation } \\
\mathrm{N} \text {, group } \mathrm{N} \text {, total }(\%)\end{array}$ & $\begin{array}{l}\text { After implementation } \\
\mathrm{N} \text {, group/ } \mathrm{N} \text {, total }(\%)\end{array}$ & $\mathrm{P}^{*}$ \\
\hline Overall & $881(100.0)$ & $827(100.0)$ & \\
\hline Term newborns & $778(88.3)$ & $727(87.9)$ & 0.798 \\
\hline Preterm newborns & $103(11.7)$ & $100(12.1)$ & \\
\hline \multicolumn{4}{|c|}{ Healthcare utilization related to suspected EOS } \\
\hline \multicolumn{4}{|c|}{ Empiric antibiotics } \\
\hline Overall & 100/881 (11.4) & $50 / 827(6.0)$ & $<0.001$ \\
\hline Term newborns & $85 / 778(10.9)$ & $46 / 727(6.3)$ & 0.001 \\
\hline \multirow[t]{2}{*}{ Preterm newborns } & $15 / 103(14.6)$ & $4 / 100(4.0)$ & 0.009 \\
\hline & Mean (SD) & Mean (SD) & \\
\hline \multicolumn{4}{|l|}{ Length of stay in days } \\
\hline Overall & $3.48(4.16)$ & $3.27(3.78)$ & 0.281 \\
\hline Term newborns & $2.95(2.97)$ & $2.58(1.96)$ & 0.005 \\
\hline Preterm newborns & $7.48(7.98)$ & $8.27(7.88)$ & 0.475 \\
\hline \multicolumn{4}{|c|}{ EOS-related laboratory tests } \\
\hline Overall & $2.34(4.77)$ & $1.63(3.62)$ & $<0.001$ \\
\hline Term newborns & $2.08(4.28)$ & $1.42(3.42)$ & $<0.001$ \\
\hline Preterm newborns & $4.32(7.24)$ & $3.16(4.57)$ & 0.173 \\
\hline \multicolumn{4}{|c|}{ Antibiotic days for suspected EOS } \\
\hline Overall & $0.57(1.84)$ & $0.36(1.47)$ & 0.009 \\
\hline Term newborns & $0.57(1.85)$ & $0.37(1.85)$ & 0.023 \\
\hline Preterm newborns & $0.55(1.77)$ & $0.24(1.23)$ & 0.144 \\
\hline \multicolumn{4}{|c|}{ Financial costs related to suspected EOS } \\
\hline \multicolumn{4}{|c|}{ Costs associated with length of stay, in $€$} \\
\hline Overall & $2614(3034)$ & $2516(2737)$ & 0.481 \\
\hline Term newborns & $2215(2141)$ & 2019 (1444) & 0.03 \\
\hline Preterm newborns & $5629(5842)$ & $6128(5676)$ & 0.537 \\
\hline \multicolumn{4}{|c|}{ Costs associated with EOS-related laboratory tests, in $€$} \\
\hline Overall & $36.8(89.5)$ & $24.9(59.2)$ & $<0.001$ \\
\hline Term newborns & $31.4(75.6)$ & $21.0(54.7)$ & 0.002 \\
\hline Preterm newborns & $77.7(154)$ & $52.7(79.8)$ & 0.147 \\
\hline \multicolumn{4}{|c|}{ Cost associated with antibiotic treatment, in $€$} \\
\hline Overall & $1.54(5.13)$ & $0.96(3.99)$ & 0.008 \\
\hline Term newborns & $1.56(5.17)$ & $1.00(4.08)$ & 0.020 \\
\hline Preterm newborns & $1.45(4.80)$ & $0.64(3.23)$ & 0.164 \\
\hline \multicolumn{4}{|l|}{ Combined costs, in $€$} \\
\hline Overall & $2653(3092)$ & $2542(2772)$ & 0.434 \\
\hline Term newborns & $2248(2190)$ & $2041(1480)$ & 0.020 \\
\hline Preterm newborns & $5708(5940)$ & $6181(5731)$ & 0.564 \\
\hline
\end{tabular}

*Welch two-sample t-test

Our findings of reduced economic costs in term newborns align with a recent theoretical study by Gong et al., predicting significant costs reductions due to EOS calculator implementation [7]. For acute medical care, the model by Gong et al. predicted estimated cost savings of $1930 \$$, equaling a relative reduction of 52\%. Mean cost reduction for term newborns in our study was significantly smaller, at $207 €$ or a relative reduction of $9 \%$. This may be explained by several factors. First,
Gong et al. used a fictitious relative reduction of $67 \%$ in empiric antibiotic treatment by implementation of the EOS calculator, which is significantly above real-world evidence in the literature [4]. Second, the predicted cost savings were based on American healthcare costs, which are relatively high compared with European countries [16].

Finally, earlier studies reporting significant reductions in hospitalizations and other secondary benefits were performed 


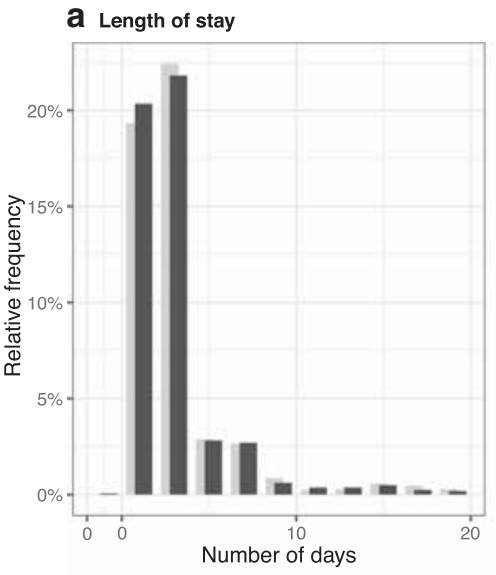

b EOS-related laboratorium tests

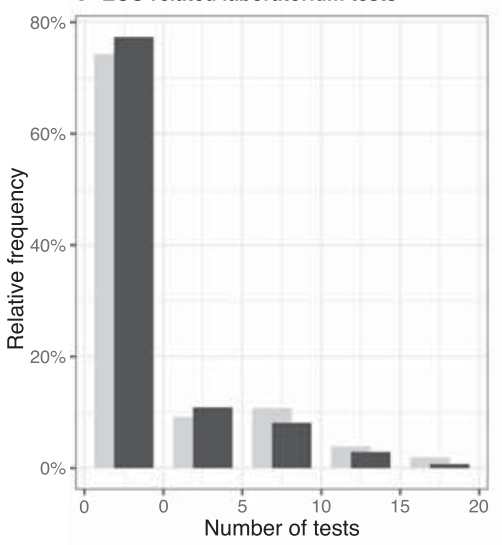

C Antibiotic days

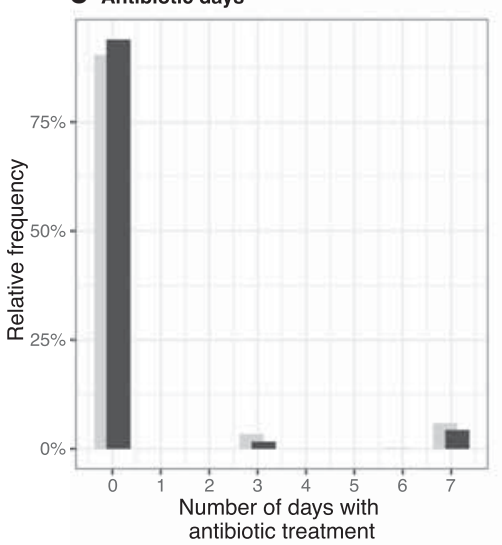

Cohort

Before implementation

After implementation d Costs associated with length of stay

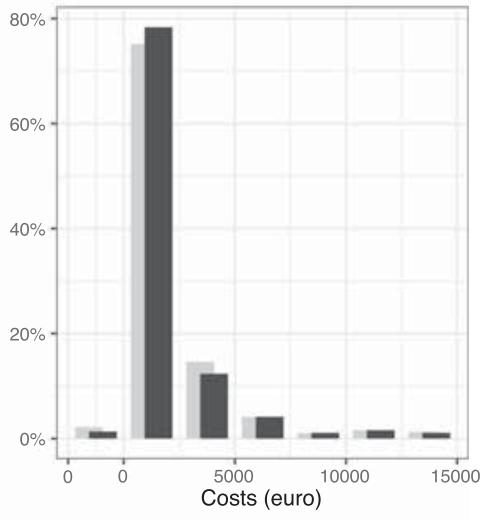

Costs associated with

EOS-related laboratorium tests

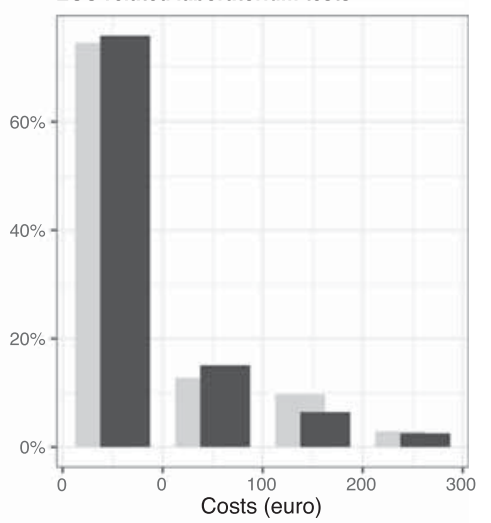

f Costs associated with

antibiotics for suspected EOS

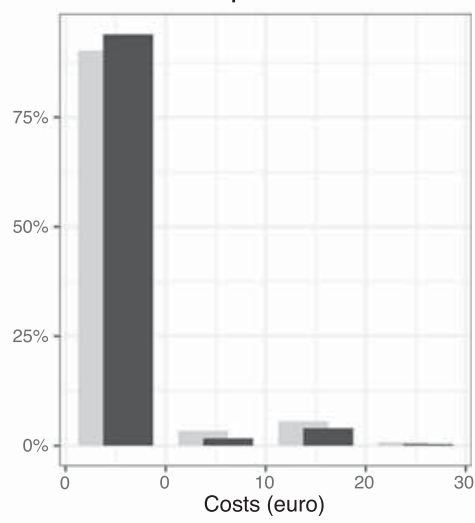

Costs (euro)

Combined costs associated with suspected EOS

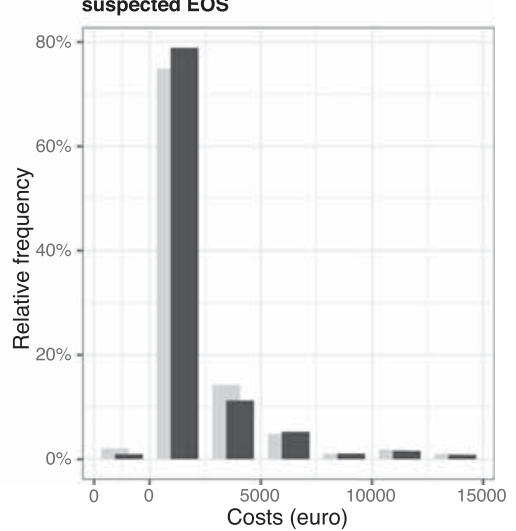


Fig. 2 EOS-related healthcare utilization and associated costs before and after implementation of the EOS calculator. Distributions of the frequencies of clinical outcomes (panels A, B, and C) and associated costs (panels D through G), for cohorts before and after implementation. Frequency of zero as a value displayed as the first bin in continuous variables (panels $\mathbf{A}, \mathbf{B}$, and $\mathbf{D}$ through $\mathbf{G}$ ). Outliers omitted from panels (A, $n=23 ; \mathbf{B}, n=9 ; \mathbf{C}, n=3 ; \mathbf{D}, n=20 ; \mathbf{E}, n=25, \mathbf{F}, n=1$; $\mathbf{G}, n=24)$ for optical clarity; no outliers were removed from analysis

in populations with relative high rates of neonatal ward hospitalization among well-appearing newborns and use of blood cultures without start of empiric antibiotic treatment [2]. Both of these practices are uncommon in European settings, including ours [17-19].

Strengths of this novel study include the use of robust data from electronic hospital registration systems for clinical and economical outcomes and for an unbiased determination of eligibility of patients. We included data from all admitted newborns to avoid selection bias when selecting at-risk newborns. Because the EOS calculator was applied only when a newborn was considered at-risk based on maternal risk factors or clinical symptoms, this means our results may underestimate cost reductions on the patient level associated with the EOS calculator. Although the study is inherently limited by its retrospective and temporal nature, our results are corrected for temporal cost changes, and data were available for all included newborns. Finally, our study used real-world billing costs for cost calculations, specific for our center. Different applicable costs in other centers and countries will impact the size of cost reductions associated with EOS calculator implementation.

To our knowledge, this is the first study to evaluate the effects of implementation of the EOS calculator on healthcare utilization and financial costs using non-hypothetical data

Table 2 EOS care utilization and associated costs in at-risk newborns with or without empirical antibiotics for suspected EOS

\begin{tabular}{|c|c|c|c|}
\hline & $\begin{array}{l}\text { Treated } \\
\text { with AB } \\
(\mathrm{n}=150)\end{array}$ & $\begin{array}{l}\text { Not treated } \\
\text { with } A B \\
(n=1558)\end{array}$ & $\mathrm{P}^{*}$ \\
\hline & Mean (SD) & Mean (SD) & \\
\hline Length of stay, in days & $7.37(4.88)$ & $2.99(3.66)$ & $<0.001$ \\
\hline $\begin{array}{l}\text { Number of EOS-related } \\
\text { laboratory tests }\end{array}$ & $11.2(4.71)$ & $1.11(2.99)$ & $<0.001$ \\
\hline $\begin{array}{l}\text { No. of days with } \mathrm{AB} \\
\text { for suspected EOS }\end{array}$ & $5.29(2.54)$ & $0.00(0.00)$ & $<0.001$ \\
\hline $\begin{array}{l}\text { Costs associated with } \\
\text { duration of hospital stay }\end{array}$ & $5492(3587)$ & $2285(2655)$ & $<0.001$ \\
\hline $\begin{array}{l}\text { Costs associated with } \\
\text { EOS-related laboratory tests }\end{array}$ & $194(117)$ & $15.3(47.9)$ & $<0.001$ \\
\hline $\begin{array}{l}\text { Cost associated with } \\
\text { antibiotic treatment } \\
\text { for suspected EOS }\end{array}$ & $14.3(7.46)$ & $0.00(0.00)$ & $<0.001$ \\
\hline Combined costs & $5700(3639)$ & $2300(2684)$ & $<0.001$ \\
\hline
\end{tabular}

*Welch two-sample t-test from implementing the calculator in daily clinical practice. Its findings suggest that the benefits of the EOS calculator are predominantly clinical, including decreased unnecessary treatment and fewer laboratory tests. In addition, we found significant reductions in duration of hospital admission and economic costs for term newborns at risk for EOS, further reducing the burden of suspected EOS. The economic benefits will depend on healthcare tariffs and clinical protocols of a particular setting. However, the clinical benefits may very well justify implementation of the EOS calculator, even if economic benefits are modest.

\section{Conclusion}

In addition to the well-known positive impact on antibiotic stewardship, implementation of the EOS calculator is also clearly associated with reductions in the healthcare utilization related to suspected EOS in late preterm and term newborns and with a reduction in associated financial costs among those born term.

Acknowledgments We are grateful to Juliette Hooghiemstra, BSc (Vrije Universiteit, Amsterdam), and the Departments of Clinical Pharmacy and Finance and Control of Tergooi Hospital for their essential assistance in data collection.

Authors' contribution NBA and FBP designed the study. NBA, DHV, ET, WG, JBvG, FPB, helped to draft the manuscript. NBA, DHV, ET, WG, JBvG, FPB read and approved the final manuscript.

\section{Compliance with ethical standards}

Conflict of interest The authors declare that they have no conflict of interest.

Ethical standards This article does not contain any studies with human participants or animals performed by any of the authors. This study was approved by the Scientific Review Committee of Tergooi Hospitals (study number 15.58; letter reference kV/15.69).

Informed consent Informed consent by patients and caregivers was not required.

Open Access This article is distributed under the terms of the Creative Commons Attribution 4.0 International License (http:// creativecommons.org/licenses/by/4.0/), which permits unrestricted use, distribution, and reproduction in any medium, provided you give appropriate credit to the original author(s) and the source, provide a link to the Creative Commons license, and indicate if changes were made.

\section{References}

1. Escobar GJ, Puopolo KM, Wi S, Turk BJ, Kuzniewicz MW, Walsh EM, Newman TB, Zupancic J, Lieberman E, Draper D (2014) Stratification of risk of early-onset sepsis in newborns $\geq 34$ weeks' 
gestation. Pediatrics 133:30-36. https://doi.org/10.1542/peds.20131689

2. Kuzniewicz MW, Puopolo KM, Fischer A, Walsh EM, Li S, Newman TB, Kipnis P, Escobar GJ (2017) A quantitative, riskbased approach to the management of neonatal early-onset sepsis. JAMA Pediatr 171:365-371. https://doi.org/10.1001/ jamapediatrics.2016.4678

3. Achten NB, Dorigo-Zetsma JW, van der Linden PD, van Brakel M, Plötz FB (2018) Sepsis calculator implementation reduces empiric antibiotics for suspected early-onset sepsis. Eur J Pediatr 177:741746. https://doi.org/10.1007/s00431-018-3113-2

4. Achten NB, Klingenberg C, Benitz WE, Stocker M, Schlapbach LJ, Giannoni E, Bokelaar R, Driessen GJA, Brodin P, Uthaya S, van Rossum AMC, Plötz FB (2019) Association of use of the neonatal early-onset sepsis calculator with reduction in antibiotic therapy and safety: a systematic review and meta-analysis. JAMA Pediatr 173: 1032. https://doi.org/10.1001/jamapediatrics.2019.2825

5. Beavers JB, Bai S, Perry J et al (2018) Implementation and evaluation of the early-onset sepsis risk calculator in a high-risk university nursery. Clin Pediatr (Phila) 57:1080-1085. https://doi.org/10. 1177/0009922817751337

6. Gievers LL, Sedler J, Phillipi CA, Dukhovny D, Geddes J, Graven P, Chan B, Khaki S (2018) Implementation of the sepsis risk score for chorioamnionitis-exposed newborns. J Perinatol 38:1-1587. https://doi.org/10.1038/s41372-018-0207-7

7. Gong CL, Dasgupta-Tsinikas S, Zangwill KM, Bolaris M, Hay JW (2019) Early onset sepsis calculator-based management of newborns exposed to maternal intrapartum fever: a cost benefit analysis. J Perinatol 39:571-580. https://doi.org/10.1038/s41372-019-0316y

8. Ayrapetyan M, Carola D, Lakshminrusimha S et al (2018) Infants born to mothers with clinical chorioamnionitis: a cross-sectional survey on the use of early-onset sepsis risk calculator and prolonged use of antibiotics. Am J Perinatol 1. https://doi.org/10.1055/s-00381668548

9. Dhudasia MB, Mukhopadhyay S, Puopolo KM (2018) Implementation of the sepsis risk calculator at an academic birth hospital. Hosp Pediatr 8:243-250. https://doi.org/10.1542/hpeds. 2017-0180

10. Strunk T, Buchiboyina A, Sharp M, Nathan E, Doherty D, Patole S (2018) Implementation of the neonatal sepsis calculator in an Australian tertiary perinatal centre. Neonatology 113:379-382. https://doi.org/10.1159/000487298
11. American Academy of Pediatrics Committee on Fetus and Newborn (2012) Levels of neonatal care. Pediatrics 130:587-597. https://doi.org/10.1542/peds.2012-1999

12. Skovlund E, Fenstad GU (2001) Should we always choose a nonparametric test when comparing two apparently nonnormal distributions? J Clin Epidemiol 54:86-92

13. Fagerland MW, Sandvik L (2009) Performance of five two-sample location tests for skewed distributions with unequal variances. Contemp Clin Trials 30:490-496. https://doi.org/10.1016/j.cct. 2009.06.007

14. Fagerland MW (2012) T-tests, non-parametric tests, and large studies - a paradox of statistical practice? BMC Med Res Methodol 12: 78. https://doi.org/10.1186/1471-2288-12-78

15. Mukherjee A, Davidson L, Anguvaa L, Duffy DA, Kennea N (2015) NICE neonatal early onset sepsis guidance: greater consistency, but more investigations, and greater length of stay. Arch Dis Child Fetal Neonatal Ed 100:F248-F249. https://doi.org/10.1136/ archdischild-2014-306349

16. Papanicolas I, Woskie LR, Jha AK (2018) Health care spending in the United States and other high-income countries. JAMA - J Am Med Assoc 319:1024-1039. https://doi.org/10.1001/jama.2018. 1150

17. National Institute for Health and Clinical Excellence (2012) Neonatal infection (early onset): antibiotics for prevention and treatment. In: Clin. Guidel. https://www.nice.org.uk/guidance/ cg149/resources/neonatal-infection-early-onset-antibiotics-forprevention-and-treatment-35109579233221. Accessed 19 Jun 2018

18. NVOG (Nederlandse Vereniging voor Obstetrie en Gynaecologie), NVK (Nederlandse Vereniging Kindergeneeskunde) (2017) Preventie en behandeling van early-onset neonatale infecties (Adaptatie van de NICE-richtlijn). 1-94

19. van Herk W, el Helou S, Janota J, Hagmann C, Klingenberg C, Staub E, Giannoni E, Tissieres P, Schlapbach LJ, van Rossum A, Pilgrim SB, Stocker M (2016) Variation in current management of term and late-preterm neonates at risk for early-onset sepsis: an international survey and review of guidelines. Pediatr Infect Dis J 35:494-500. https://doi.org/10.1097/INF.0000000000001063

Publisher's note Springer Nature remains neutral with regard to jurisdictional claims in published maps and institutional affiliations. 